\title{
Thom H. Dunning, Jr.: Contributions to chemical theory and computing
}

\author{
Angela K. Wilson · Kirk A. Peterson • David E. Woon
}

Published online: 27 December 2014

(C) Springer-Verlag Berlin Heidelberg 2014

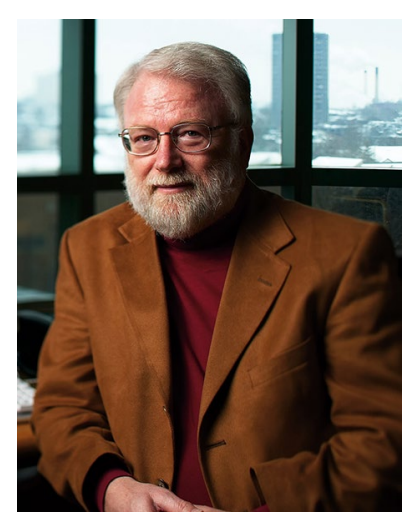

Thom H. Dunning, Jr., provides an outstanding example of success in science, computing, and scientific leadership. From his scientific contributions to knowledge to his developments and accomplishments that have enabled discovery, Thom's influence upon science, computing, and the scientific community has been significant.

Published as part of the special collection of articles celebrating the career of Professor Thom Dunning upon his retirement.

A. K. Wilson $(\square)$

Department of Chemistry, University of North Texas, 1155 Union Circle, \#305070, Denton, TX 76203-5017, USA

e-mail: akwilson@unt.edu

\section{K. A. Peterson}

Department of Chemistry, Washington State University, Pullman, WA 99164-4630, USA

e-mail: kipeters@wsu.edu

\section{E. Woon}

Department of Chemistry, University of Illinois

Urbana-Champaign, Urbana, IL 61801, USA

e-mail: davidewoon@gmail.com

As a chemistry undergraduate at the Missouri University of Science and Technology, which was then called the University of Missouri at Rolla, Thom was fascinated by the ability of organic chemists to rationalize the course of a broad range of chemical reactions by shuttling electrons around in the molecules. However, he was also somewhat skeptical-this seemed too good to be true. The following year he took a course in quantum mechanics in the Physics Department and realized that quantum mechanics was the means for fact-checking the explanations in organic chemistry. Thus began his passion for chemical theory. In the quantum mechanics course, he encountered the differential equation for the states and wavefunctions of the harmonic oscillator. Since he had just had a course in numerical analysis and the university was touting its new, "very powerful" computer that it had just installed (an IBM 1620!), Thom decided to solve the harmonic oscillator equation on the computer. After teaching himself the new programming language developed by IBM-FORTRAN—and coding the problem, he was amazed to find that his numerical solution agreed perfectly with that obtained analytically. Thus began his passion for computing.

\section{Contributions to chemical theory}

Thom's research career has focused on topics in chemical theory (e.g., open-shell Hartree-Fock theory, generalized valence bond theory), numerical techniques for solving the Schrödinger equation (e.g., basis sets for both orbital and correlated calculations), and applications of computation to important chemistry problems (molecular physics of lasers, species and reactions involved in combustion, structure and energetics of aqueous clusters, and, more recently, the unusual chemistry of the late p-block elements in the second 
row and beyond). Below is a brief summary of Thom's contributions to chemical theory and computation.

\subsection{Generalized valence bond theory}

Thom began his graduate career at Caltech in 1965, just as Dr. William A. Goddard III accepted an appointment as an Arthur Amos Noyes Fellow in the Chemistry Division. Although Bill could not accept students at that point, Thom was impressed by the insights provided by the valence bondbased theory that he was developing (later called generalized valence bond, GVB, theory). The GVB wavefunction corrects the main deficiencies of the Hartree-Fock/molecular orbital wavefunction while providing a conceptually appealing, chemistry-oriented description of the electronic structure of molecules. Although Thom's thesis research focused on the excited states of the ethylene molecule with Professor B. Vincent McKoy, as a postdoctoral fellow, he contributed to the development of the perfect pairing, strong orthogonality version of GVB theory. Imposition of these constraints made it possible to extend GVB calculations to a broad range of molecules, often with only modest sacrifices in accuracy. A paper summarizing early work in this area was published in Accounts of Chemical Research (Goddard et al., Acc. Chem. Res. 1973, 6, 368-376).

More recently, Dr. Dunning, Dr. David E. Woon, and the rest of his research group at the University of Illinois at Urbana-Champaign used the GVB method, as well as more sophisticated coupled cluster and multireference configuration interaction methods, to study the electronic structure of hypervalent molecules, e.g., $\mathrm{PF}_{5}, \mathrm{SF}_{4} / \mathrm{SF}_{6}$, and $\mathrm{ClF}_{3} / \mathrm{ClF}_{5}$. It was found that a new type of bond, the recoupled pair bond, provides the basis for the formation of hypervalent molecules. In fact, the ability of the second row elements to form this new type of bond explains many of the other anomalies associated with the chemistry of the late p-block elements in the second row and beyond: new bound low-lying excited states, edge versus vertex inversion in heavily halogenated species, and unusually rapid chemical reactions to name just a few of these anomalies. A paper summarizing early work in this area was published in Accounts of Chemical Research (Dunning et al., Acc. Chem. Res. 2013, 46, 359-368). These full GVB calculations were made possible by the CASVB program, which was developed by T. Thorsteinsson and D. Cooper and is included in both the MOLPRO (http://www.molpro.net/) and MOLCAS (http://www.molcas.org/) electronic structure packages.

\subsection{Basis sets for molecular computations}

Dr. Dunning is perhaps best known for the development of basis sets for use in molecular calculations. In graduate school, Thom found the existing Gaussian basis sets for Hartree-Fock calculations on molecules of interest to him-ethylene, formaldehyde, and other organic molecules-to be lacking. Thom systematically explored the construction of new basis sets, laying out a set of rules that enabled the construction of high-quality basis sets for Hartree-Fock calculations. In the late 1980s, at Argonne National Laboratory, he became frustrated at the inability to converge configuration interaction calculations with regard to the basis set. Then Jan Almlöf and Peter Taylor reported that the occupations of the natural orbitals from atomic calculations grouped naturally into sets with similar occupation numbers. Thom had no explanation for this finding and set out on a systematic exploration of correlation in atoms. The result of this study was the development of the correlation-consistent basis sets for the first row atoms $(\mathrm{H}, \mathrm{He}, \mathrm{B}-\mathrm{Ne})$, a family of basis sets that provide systematic, increasingly accurate solutions of the electronic Schrödinger equation for atoms and molecules. After his move to Pacific Northwest National Laboratory, this work was extended to other elements of the Periodic Table and to the use of these basis sets to quantify the intrinsic error in electronic structure methods (see following paragraph). These basis sets are used in almost all computational chemistry codes. Six scientific publications in this series are "Citation Classics," i.e., they have received more than 1000 citations (J. Chem. Phys. 1970, 53, 2823-2833; ibid. 1971, 55, 716-723; ibid. 1989, 90, 1007-1023; ibid. 1992, 96, 6796-6806; ibid. 1993, 98, 1358-1371; ibid. 1995, 103, 4572-4585). This work was summarized in a chapter in the Encyclopedia of Computational Chemistry (Dunning et al., Encyclopedia of Computational Chemistry, Ed. P. v. R. Schleyer, John Wiley \& Sons Ltd, 1997).

\subsection{Assessment of electronic structure methods}

The development of the correlation-consistent basis sets, which systematically approach the complete basis set limit, made it possible to separate the basis set error from the error due to the electronic structure method. This made it possible to determine the intrinsic error associated with a given electronic structure method (configuration interaction, perturbation theory, coupled cluster, etc.). Dr. Dunning and his co-workers used this approach to rigorously assess the accuracy of the quantum chemical methodologies used to describe the electronic structure of molecules. This work yielded a number of major surprises, e.g., the poor/non-convergence of the perturbation expansion for a broad range of molecules, and established the intrinsic accuracy of the coupled cluster method. A "Feature Article" summarizing some of this work was published in the Journal of Physical Chemistry A (T. H. Dunning, Jr., J. Phys. Chem. A 2000, 104, 9062-9080). 


\subsection{Characterization of molecular lasers}

At Los Alamos National Laboratory, Dr. Dunning and Dr. P. Jeffrey Hay were involved in characterizing the electronic states responsible for laser action in a number of exotic molecular systems (rare has halides, rare gas oxides, etc.), which were being developed for laser fusion and laser isotope separation applications. These molecules have unstable ground states and, thus, are very difficult to characterize experimentally. The computational studies provided information on these molecules (e.g., excited state lifetimes) that were otherwise unavailable and guided subsequent experimental studies of these species. As a result of the computational studies, a new laser transition in the rare gas halides was predicted and later observed. A review article describing this work was published in the Annual Reviews of Physical Chemistry (P. J. Hay, T. H. Dunning, Jr. and W. R. Wadt, Annu. Rev. Phys. Chem. 1979, 30, 311-346).

\subsection{Chemical species and reactions in combustion}

When Dr. Dunning moved from Los Alamos National Laboratory to Argonne National Laboratory, he led a research group focused on characterizing the molecular species and reactions involved in the combustion of hydrogen and hydrocarbon fuels. These studies provide new insights into the chemical reactions involved in flames, answering some questions that had puzzled chemical kineticists and combustion modelers for decades. This work also provided information on the complex pathways involved in the reactions of hydrocarbon species, including a wealth of heretofore unavailable information on reaction products and branching ratios. Using the information on quantum chemical methods/basis sets referred to above developed the protocol to compute the heats of many chemical reactions to an accuracy that is comparable to (and often better than) that obtained from experiment.

\section{Contribution to computing}

Thom's leadership abilities were recognized early on when he was appointed Associate Group Leader for Molecular Theory in the Laser Theory Group at Los Alamos National Laboratory. With the untimely passing of Chris Wahl, Thom was recruited to Argonne National Laboratory, where he was appointed the Group Leader of the Theoretical and Computational Chemistry Group. During Thom's tenure at Argonne, the group became an international focal point for theoretical and computational studies of the species and reactions involved in combustion. The success of this effort led to Thom's recruitment to the Pacific Northwest National Laboratory, which was planning the development of a new molecular science research center-an activity that eventually matured into the Environmental Molecular Sciences Laboratory (EMSL), a $\$ 230$ million project funded by the Office of Biological and Environmental Research (BER) in the US Department of Energy (DOE). Although the EMSL project was funded by BER, it also had strong support and guidance from Dr. Robert S. Marianelli in the Chemical Sciences Division in the Office of Basic Energy Sciences.

The EMSL Project began just as computing technologies were transitioning from the fast vector supercomputers built by Cray Research to "massively" parallel computers based on microprocessors. The trouble was that to take full advantage of these new computers, the codes used to model molecular systems had to be rewritten to distribute the compute load over the processors. This was a major undertaking. Fortunately, the EMSL project provided the funding to do this, and Thom recruited a top team of computational chemists, including Robert Harrison, Jeff Nichols, and Ricky Kendall as well as computer scientists and applied mathematicians to tackle this problem. This effort led to the development of NWChem, an application that has maintained its position at the leading edge of parallel chemistry software for more than 15 years and has scaled from the 100's of processors available in the mid-1990s to the 100,000 's of cores available today.

The success of the NWChem project led the US DOE to ask Thom to help them plan a new DOE-wide initiative in scientific simulation. Although they stated this would only be a temporary assignment, Thom was, in fact, the Assistant Director for Scientific Simulation in DOE's Office of Science for more than 2 years. The culmination of this effort was DOE's "Scientific Discovery through Advanced Computing" (SciDAC) program, which brought together disciplinary computational scientists, computer scientists, and applied mathematicians to enable a broad range of scientific computational disciplines-from biology and chemistry through materials science to fusion energy, nuclear physics and particle physics - to take full advantage of parallel computers, which at the end of the 1990s were capable of executing one trillion arithmetic operations per second (a teraflop) to a quadrillion operations per second (a petaflop) by the end of the 2000s.

After Thom left his assignment at the Office of Science, he briefly held the position of Vice President for High Performance Computing \& Communications at MCNC and was responsible for high performance computing and networking for the University of North Carolina System as well as Duke University and Wake Forest University. He was then appointed the founding director of the Joint Institute for Computational Sciences, Distinguished Professor of Chemistry and Chemical Engineering at the University of Tennessee, and Distinguished Scientist at Oak Ridge National Laboratory. In 2004, he accepted the position of 
director of the National Center for Supercomputing Applications and Distinguished Chair for Research Excellence in the Department of Chemistry at the University of Illinois at Urbana-Champaign. In October 2006, Dr. Dunning was also appointed the founding director of a new Institute for Advanced Computing Applications and Technology at UIUC. This Institute has a charter similar to that of UIUC's Beckman Institute, namely, to foster multi- and interdisciplinary research, in this case, at the interface between disciplinary computational science, computer science, and applied mathematics.

As NCSA director, Dr. Dunning led the effort to obtain funding for and then the deployment of the largest supercomputer system ever to be sited at a university-the Blue Waters Sustained Petascale Computer. This \$208 million project for the National Science Foundation was visionary in that it clearly defined a computing system that would enable the computational science and engineering community to make a major advance in their research. The project not only developed the specifications for the computer system, it also established a technical user assistance program to ensure that the research teams granted access to Blue Waters could take full advantage of its capabilities. Also during Thom's tenure, NCSA was selected to lead NSF's national cyberinfrastructure project-XSEDE, the Extreme Science \& Engineering Discovery Environment. NCSA is now widely recognized as one of the world's preeminent supercomputing centers and the only one of its kind on a university campus anywhere in the world.

At the end of 2013, Thom retired from the University of Illinois at Urbana-Champaign. But, retirement does not mean that he has lost interest in chemistry and computing. As soon as he had retired from UIUC, Thom accepted a part-time position with the Pacific Northwest National Laboratory to help PNNL and the University of Washington establish the new Northwest Institute for Advanced Computing. He was also appointed a Distinguished Faculty Fellow in UW's Department of Chemistry. We look forward to Thom's continuing contributions to the field.

Thom's recognitions include the American Chemical Society Award for Computers in Chemical and Pharmaceutical Research (2011), Distinguished Associate Award (2001) and E. O. Lawrence Award in Chemistry (1996) from the U.S. DOE, Award for Excellence in Technology Transfer (2000) from the Federal Laboratory Consortium for Technology Transfer, Fellow of the American Chemical Society (2011), Fellow of the American Physical Society (1992), and Fellow of the American Association for the Advancement of Science (1992).

Angela K. Wilson, Kirk A. Peterson, and David E. Woon 